\title{
Pharmacokinetics and metabolism of rectally administered paracetamol in preterm neonates
}

\author{
R A van Lingen, J T Deinum, J M E Quak, A J Kuizenga, J G van Dam, K J S Anand, \\ D Tibboel, A Okken
}

Department of

Paediatrics,

Division of

Neonatology,

Sophia Hospital

PO Box 10400

8000 GK Zwolle,

The Netherlands

$R$ A van Lingen

J M E Quak

Department of

Hospital Pharmacy

J T Deinum

A J Kuizenga

J G van Dam

Department of Paediatrics, Arkansas Children's Hospital and University of

Arkansas for Medical

Sciences, Little Rock,

AR, USA

K J S Anand

Department of

Paediatric Surgery,

Sophia Children's

Hospital,

Rotterdam, The

Netherlands

D Tibboel

Department of

Paediatrics,

Wilhelmina Children's

Hospital,

Utrecht, The

Netherlands

A Okken

Presented in part at the annual meeting of the European Society for

Pediatric Research,

Rotterdam, The

Netherlands, July 3-6

1994.

Correspondence to: Dr R A van Lingen.

Accepted 7 August 1998

\begin{abstract}
Aim-To investigate the pharmacokinetics, metabolism, and dose-response relation of a single rectal dose of paracetamol in preterm infants in two different age groups.

Methods-Preterm infants stratified by gestational age groups 28-32 weeks (group 1) and 32-36 weeks (group 2) undergoing painful procedures were included in this study. Pain was assessed using a modified facies pain score.

Results-Twenty one infants in group 1 and seven in group 2 were given a single rectal dose of $20 \mathrm{mg} / \mathrm{kg}$ body weight. Therapeutic concentrations were reached in $16 / 21$ and $1 / 7$ infants in groups 1 and 2, respectively. Peak serum concentrations were significantly higher in group 1. Median time to reach peak concentrations was similar in the two groups. As serum concentration was still in the therapeutic range for some infants in group 1 , elimination half life $\left(T_{1 / 2}\right)$ could not be determined in all infants: $T_{1 / 2}$ was $11.0 \pm 5.7$ in 11 infants in group 1 and $4.8 \pm 1.2$ hours in group 2 . Urinary excretion was mainly as paracetamol sulphate. The glucuronide:sulphate ratio was $0.12 \pm 0.09$ (group 1) and $0.28 \pm 0.35$ (group $2)$. The pain score did not correlate with therapeutic concentrations.

Conclusions-A $20 \mathrm{mg} / \mathrm{kg}$ single dose of paracetamol can be safely given to preterm infants in whom sulphation is the major pathway of excretion. Multiple doses in 28-32 week old neonates would require an interval of more than 8 hours to prevent progressively increasing serum concentrations.
\end{abstract}

(Arch Dis Child Fetal Neonatal Ed 1999;80:F59-F63)

Keywords: paracetamol; pharmacokinetics; pain score; metabolism

Even preterm neonates feel pain. ${ }^{1}$ Soon after birth, many painful procedures may be required for routine neonatal intensive care, and there are few data on the use of analgesics in preterm infants. ${ }^{2}$ In contrast to the use of paracetamol (acetaminophen, APAP) in term neonates, ${ }^{3}$ the use of APAP in the first weeks of life in preterm neonates is controversial, because its pharmacokinetics, pharmacodynamics, and metabolism in this age group remain unknown. Safety concerns may prevent its use in critically ill preterm neonates.

This study aimed to investigate pharmacokinetic variables after a single dose of rectally administered APAP in preterm infants, and to investigate whether there are any age dependent differences and any dose-response correlations between serum APAP concentrations and responses to pain during painful procedures.

\section{Methods}

Twenty eight inborn neonates who were admitted to the neonatal intensive care unit were included in the study. Entry criteria were the need for insertion of arterial and venous catheters or other painful procedures, such as insertion of a chest drain. The study protocol was approved by the ethical review committee and informed parental consent was obtained. As pharmacokinetics and drug metabolism change during the last three months of gestation, ${ }^{5-6}$ and pain sensitivity may be altered after 32 weeks, ${ }^{7}$ the neonates were stratified into two gestational age groups: $28-32$ weeks (group 1) and 32-36 weeks (group 2). The gestational age of the neonates was estimated from maternal menstrual history, by routine ultrasound examination during pregnancy, and from postnatal physical characteristics (Farr score).$^{8}$ Patients were excluded if they had congenital anomalies, if the mother received tocolysis with indomethacin, or if she had been given analgesics (other than local analgesia) within 24 hours before delivery. None of the infants had sepsis or other congenital infections, or documented periods of shock.

The infants were studied on the first two days after birth. Nasal intubation, if necessary, was performed in the delivery room and the patient was transported to intensive care. In each patient heart rate, respiratory rate, arterial blood pressure and oxygen saturation were monitored continuously, together with rectal and peripheral temperatures. According to standard procedures in intensive care, umbilical venous and arterial access was obtained, and parenteral nutrition was started within 24 hours of birth.

All patients were given APAP rectally after arrival in intensive care, within one hour of birth, the dose used being as close to $20 \mathrm{mg} / \mathrm{kg}$ as the available strengths of suppository $(20 \mathrm{mg}$ for birthweights $750-1249 \mathrm{~g}, 30 \mathrm{mg}$ for $1250-$ $1749 \mathrm{~g}$, and $40 \mathrm{mg}$ for 1750-2250 g) would allow. The nurses ensured that the suppository was retained. Suppositories contained 20, 30, or $40 \mathrm{mg}$ APAP (particle size $<45 \mu \mathrm{m}$ ), and hard fat (Witepsol H 15), a synthetic mixture of mono-, di-, and triglycerides of the saturated fatty acids $\mathrm{C} 10-\mathrm{C} 18$. The suppositories were prepared and analysed for APAP content and content uniformity by the quality assurance laboratory of the hospital pharmacy. 
Table 1 Demographic data of study participants

\begin{tabular}{|c|c|c|}
\hline & $\begin{array}{l}\text { Group } 128-32 \\
\text { weeks }(n=21)\end{array}$ & $\begin{array}{l}\text { Group } 2 \\
32-36 \text { weeks }(n=7)\end{array}$ \\
\hline \multicolumn{3}{|l|}{ Maternal factors } \\
\hline $\begin{array}{l}\text { HELLP/ } \\
\text { pre-eclampsia }\end{array}$ & 6 & 1 \\
\hline $\begin{array}{l}\text { pre-eciampsia } \\
\text { Solutio placenta }\end{array}$ & 1 & \\
\hline $\begin{array}{l}\text { Blood loss placenta } \\
\text { previa }\end{array}$ & 1 & 1 \\
\hline \multicolumn{3}{|l|}{$\operatorname{Sex}$} \\
\hline Male & 13 & 2 \\
\hline Female & 8 & 5 \\
\hline \multicolumn{3}{|l|}{ Method of delivery } \\
\hline Vaginal vertex & 13 & 4 \\
\hline Vaginal breech & 1 & \\
\hline Ceasarean section & 7 & 3 \\
\hline \multicolumn{3}{|l|}{ Diagnosis at admittance } \\
\hline RDS & 7 & 1 \\
\hline SGA & 5 & \\
\hline Pneumothorax & & 1 \\
\hline Asphyxia & & 2 \\
\hline Preterm birth ${ }^{\star}$ & 2 & \\
\hline Artificial ventilation & 10 & $5 \ddagger$ \\
\hline Surfactant & & \\
\hline Birthweight $(\mathrm{g}) \dagger$ & $1280(284)$ & $1786(323)$ \\
\hline $\begin{array}{l}\text { Gestational age } \\
\text { (weeks) } \dagger\end{array}$ & $30.21(0.99)$ & $33.57(0.77)$ \\
\hline
\end{tabular}

Before APAP was administered a $0.1 \mathrm{ml}$ blood sample was taken from the arterial catheter. Subsequently samples were taken at 30 , 60,120 minutes, 4,6 , and 8 hours in the first 10 neonates, with an additional sample at 12 hours in 18 neonates. After collection serum was separated and frozen at $-20^{\circ} \mathrm{C}$, until assayed. Urine was collected for 48 hours using a plastic bag, and frozen in separate (3 hours) aliquots until analysis.

The assay was performed within a month of sample collection, using a modified high performance liquid chromatography (HPLC) method. ${ }^{9}$ Serum samples were extracted with perchloric acid and after centrifugation the supernatant fluid was injected into the HPLC column. Urine samples were diluted with distilled water (1:9) before injection into the column. Standards for serum APAP, urine APAP, APAP-glucuronide (APAP-G) and APAP-sulphate (APAP-S) were injected at the start and the end of each run. HPLC conditions, retention times, and ultraviolet detection were as described before. ${ }^{9}$ Limit of detection for serum APAP was $0.2 \mathrm{mg} / 1$, recovery was $96 \%$, and precision $2 \%$. Detection limits in urine were $0.3,2.3$, and $6.3 \mathrm{mg} / 1$ for APAP, APAP-G, and APAP-S, respectively; recovery for APAP was $100 \%$, for APAP-G and APAP-S 95\%; and precision for APAP was $7 \%$, for APAP-G and APAP-S 3\%.

The values for each metabolite were converted to the equivalent weight of APAP, from which the metabolite was derived by correction for molecular weight. Standard metabolites were a generous gift from Sterling Health, Haarlem, The Netherlands. The calibration curves were linear over the range $0.5-40 \mathrm{mg} / 1$ (APAP) and 5-1000 mg/l (metabolites).

Pharmacokinetic data and variables were calculated from standard equations using the KINFIT program (Mediware, Groningen, The Netherlands). Concentration time curves were constructed to determine peak serum concen-
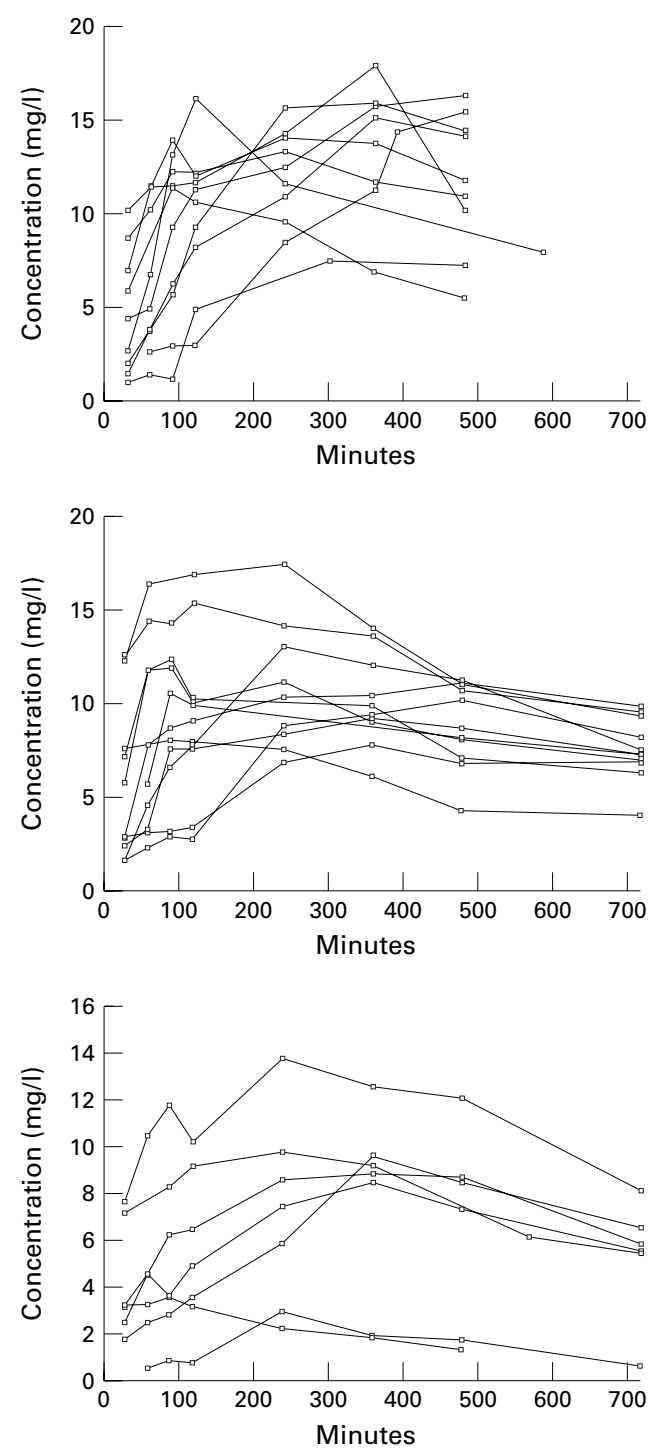

Figure 1 (A) APAP serum concentrations after a single dose of $20 \mathrm{mg} / \mathrm{kg}$, after 30 minutes to 8 hours in 10 infants (group 1); (B) after 30 minutes to 12 hours in 11 infants (group 1); and (C) after 30 minutes to 12 hours in seven infants (group 2).

tration $\left(\mathrm{C}_{\max }\right)$, time to reach $\mathrm{C}_{\max }$, time to reach therapeutic concentrations (adult values 10-20 $\mathrm{mg} / \mathrm{l}),{ }^{4}{ }^{10}$ and serum half life of the drug $\left(\mathrm{t}_{1 / 2}\right)$. For $t \frac{1 / 2}{2}$, a minimum of two time points in the elimination phase was used. Kinetic constants for APAP elimination were calculated according to the two compartment model as used by Miller. ${ }^{11}$ For calculations a bio-availability of $90 \%$ was assumed.

Because all infants were nursed in incubators with a relative ambient humidity of $60-70 \%$ and temperature settings that aimed at a neutral temperature between $36.5-37.2^{\circ} \mathrm{C}$, the anti-pyretic effects could not be measured.

Pain was assessed by nurse pairs or nurse/ doctor pairs with a modified five facies pain score, showing increasing levels of discomfort from 0 (no pain) to 4 (clearly/obviously in pain)..$^{1213}$

Data were analysed using the Statistical Package for the Social Sciences, SSPS Inc., Chicago, Ill, USA. ${ }^{14}$ Student's $t$ tests were used 
Table 2 Pharmacokinetic parameters of paracetamol in preterm neonates after rectal single dose

\begin{tabular}{llll}
\hline Parameter & $\begin{array}{l}\text { 28-32 weeks } \\
(1)\end{array}$ & $\begin{array}{l}32-36 \text { weeks } \\
(2)\end{array}$ & p Value \\
\hline $\mathrm{C}_{\max }(\mathrm{mg} / \mathrm{l})$ & $12.5(2.9)$ & $7.5(4.0)$ & 0.001 \\
$\mathrm{range}$ & $7.5-18.0$ & $1.5-13.6$ & \\
$\mathrm{~T}_{\max }(\mathrm{h})^{\star}$ & 3.9 & 5.1 & $\mathrm{NS}$ \\
$\mathrm{range}$ & $0.8-10.5$ & $1.0-9.5$ & \\
$\mathrm{~T}^{1 / 2}(\mathrm{~h})$ & $11.0(5.7)$ & $4.8(1.2)$ & 0.011 \\
$\mathrm{range}$ & $3.5-25.2$ & $3.6-6.8$ & \\
$\mathrm{AUC}(\mathrm{mg} / \mathrm{h} / \mathrm{l})$ & $95.1(28.0)$ & $71.7(41.7)$ & 0.046 \\
$\mathrm{range}$ & $29.0-160.6$ & $17.5-134.6$ & \\
$\mathrm{Cl}(1 / \mathrm{h})$ & $0.10(0.04)$ & $0.56(0.66)$ & 0.04 \\
range & $0.03-0.17$ & $0.13-1.70$ & \\
\hline
\end{tabular}

$\mathrm{C}_{\max }$, maximum concentration; $\mathrm{T}_{\max }$, time to reach maximum concentration; $\mathrm{T}^{1} 1 / 2$, elimination half life; AUC, area under the concentration-time curve; $\mathrm{Cl}$ clearance. Data are shown as mean $(\mathrm{SD})$ or as median ${ }^{\star}$

for normally distributed data and MannWhitney U tests for non-parametric data to compare the two groups. Least squares regression was used to evaluate linear correlation between variables. $P$ values of $<0.05$ (two tailed) were considered significant.

\section{Results}

Of the 28 infants studied, 21 were in the $28-32$ week group and seven in the 32-36 week group. Demographic data are shown in table 1. Serum APAP concentrations are shown in figs $1 \mathrm{~A}-\mathrm{C}$.

Analgesic therapeutic concentrations (adult values $10-20 \mathrm{mg} / \mathrm{l})^{4}{ }^{10}$ were reached in 17 infants, in 16 out of 21 in group $1(76.2 \%)$, and in one out of $7(14.3 \%)$ in group 2 . Therapeutic concentrations persisted for 8 hours in 13 infants (12 in group 1 and one in group 2) and for 12 hours in one infant. $\mathrm{C}_{\max }$ was mean 11.2 (SD 3.8) $\mathrm{mg} / \mathrm{l}$ for all infants studied; data for groups 1 and 2 are shown in table $2 ; \mathrm{C}_{\max }$ was significantly higher in group 1 . For individual values there was a significant negative correlation between $\mathrm{C}_{\max }$ and gestational age $(\mathrm{r}-0.50$, $\mathrm{p}=0.007)$. There was a significant inverse linear regression between APAP concentrations at t60 ( $<<0.05)$, t120 ( $=0.008)$, and t240 $(\mathrm{p}<0.05)$ with gestational age (fig 2) and no significant correlation between $T_{\max }$ and gestational age. As serum concentrations were still in the therapeutic range after 8 hours, $T_{1 / 2}$ could not be measured in 10 infants in group 1 because elimination of APAP was not yet apparent (fig 1A). Therefore, $\mathrm{T}_{1 / 2}$ could only be

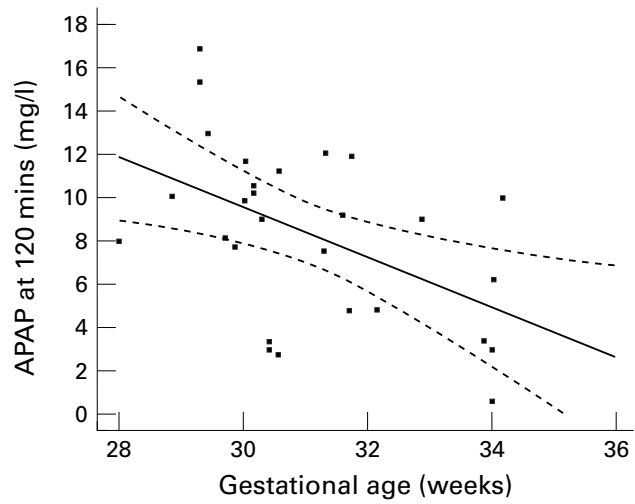

Figure 2 Linear regression analysis of gestational age on APAP concentrations at 120 minutes: $r-0.49, p 0.008$; dashed lines indicate confidence limits.

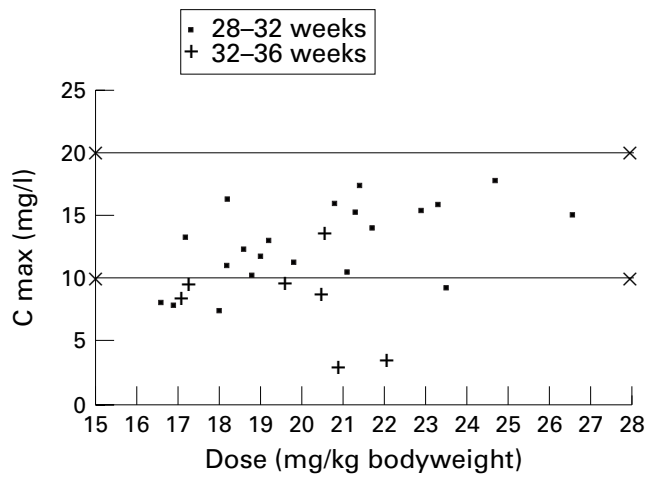

Figure 3 APAP peak serum concentrations in preterm infants in relation to dose, in two different age groups: group 128 to 32 weeks ( $n=21$ ), group 232 to 36 weeks $(n=7)$.

Therapeutic concentrations represent adult values.

measured in 11 infants in group 1 but it was measured in all infants in group 2 (table 2). In two infants only two pieces of data were available to calculate $T_{1 / 2}$; in the other 16 infants three or more time points were available. The area under the serum concentration time curve (AUC) was determined by the trapezoidal rule. The mean (SD) value in group 1 (95.1 (28.0) in 11 infants) was significantly higher than in group 2 (71.7 (41.7); p=0.046). Clearance increased with gestational age, showing a significant positive correlation $(r=0.52$, $\mathrm{p}=0.008)$ (table 2).

Due to the available strengths of the suppositories, individual doses ranged from $16.6-26.6 \mathrm{mg} / \mathrm{kg}$ in group 1 , and from $17.1-22.1 \mathrm{mg} / \mathrm{kg}$ in group 2; the relation between APAP dose and $\mathrm{C}_{\max }$ is shown in fig 3 . All doses of more than $18 \mathrm{mg} / \mathrm{kg}$ resulted in therapeutic concentrations in group 1, but not in group 2. There was a significant correlation between the rectal temperature and time to reach therapeutic concentrations ( $\mathrm{r}-0.54$, $\mathrm{p}<0.03$ ), and no correlation between rectal temperature and $\mathrm{C}_{\text {max }}$.

No significant losses occurred during urine collection. Of the rectal dose, $63.2(24.4) \%$ was recovered in the urine; $61.0(24.4) \%$ in group 1 and $74.2(24.8) \%$ in group $2(\mathrm{NS} ; \mathrm{p}=0.2)$. APAP was excreted mainly as APAP-S and to a lesser extent as APAP-G. Very small amounts of free APAP, and APAP-cysteine (APAP-C) were excreted and APAP-mercapturic acid

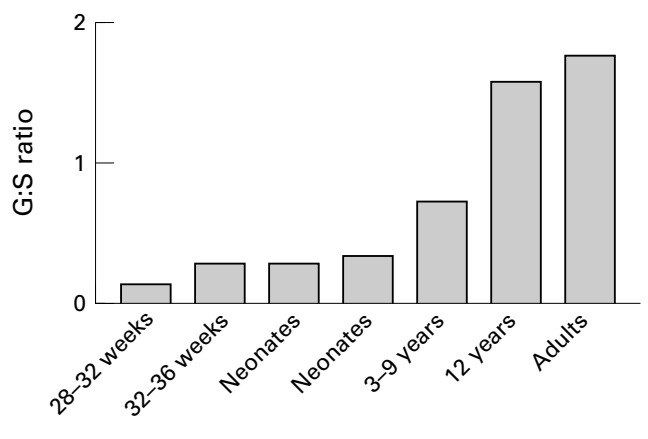

Figure 4 APAP metabolism, glucuronide:sulphate ratio in preterm infants in this study compared with term neonates ${ }^{22 \star}$ and term neonates, older children, and adults ${ }^{11}{ }^{\dagger}$ ${ }^{*}$ Reproduced by kind permission of Paediatrics and ${ }^{t}$ reproduced by kind permission of Clinical Pharmacology and Therapeutics. 
(APAP-M) was not detected. The glucuronide:sulphate ratio (G:S ratio) was $0.12(0.09)$ and $0.28(0.35)$ for groups 1 and 2, respectively (fig 4).

Facies pain score reflecting the infants' responses was scored at the time of blood sampling. Pain scores from 0 to 2 were obtained in all infants and did not correlate with therapeutic concentrations. The reliability of the pain score was high; weighted Cohen's $\kappa=0.74$.

\section{Discussion}

As far as we are aware this is the first study to provide data on the pharmacokinetics and metabolism of APAP in preterm infants. As the rectal mode of delivery results in less efficient absorption than the oral route, higher doses are needed in infants and in older children. After rectal doses of $16-26 \mathrm{mg} / \mathrm{kg}$ none of our infants reached concentrations above $20 \mathrm{mg} / \mathrm{l}$, while toxic concentrations occur above $120 \mathrm{mg} / \mathrm{l}$ four hours after ingestion. ${ }^{15}$ In infants whose mothers ingested an overdose of APAP prenatally, high concentrations of APAP (75.5 and 260 $\mathrm{mg} / \mathrm{l}$ ) were documented in neonates with no apparent hepatic or renal toxicity. ${ }^{16}{ }^{17}$ The high therapeutic ratio in neonates may be related to reduced rates of metabolism by the cytochrome p 450 system in neonatal liver and neonates' increased ability to synthesise gluthathione relative to adults. ${ }^{18}{ }^{19}$ Young mice have a fourfold greater gluthatione turnover and increased activity in the gluthatione peroxidase/reductase system than older mice. ${ }^{18}{ }^{19}$ Renal toxicity is also prevented by the increased solubility of APAP-S as compared to APAP-G, the major metabolite in adults. We speculate that multiple doses of APAP would also be safe in the preterm infants studied.

Peak serum concentrations were reached later in preterm infants in our study than in term infants and adults, ${ }^{40}$ but most $28-32$ week old infants did achieve therapeutic concentrations. All the infants in our study were under 1 hour of age, and absorption may increase with gestational and postnatal age. Individual differences in rectal temperature (range $34.7^{\circ} \mathrm{C}-38.4^{\circ} \mathrm{C}$ ) at the time of administration of the suppository may alter the time needed for melting and absorption. A significant correlation occurred between rectal temperature and the time to reach therapeutic concentrations. Rectal temperature ranged from 34.8 to 36.8 in 10 infants who did not achieve therapeutic concentrations $(>10$ $\mathrm{mg} / \mathrm{l})$. Although no suppositories were expelled, differences in $\mathrm{C}_{\max }$ and $\mathrm{T}_{\max }$ in individual patients may be due to the variability of venous drainage from the rectum. Drugs administered into the proximal rectum will be subject to the hepatic first pass effect, whereas drugs in the distal rectum will bypass the liver. ${ }^{5}$ The inverse linear regression between APAP concentrations and gestational age may be due to the larger extracellular space and thus greater volume of distribution $(\mathrm{Vd})$ with lower gestational age. ${ }^{21}$ Although our sampling time was two to four times greater than the $T_{1 / 2}$ in adults and term neonates, ${ }^{17} 22-24$ serum concentrations remained above therapeutic concentrations for more than 8 hours, which made it impossible to assess $T_{1 / 2}$ in 10 preterm infants. In infants where assessment was possible, the values indicated slow elimination, subsequent to a lower clearance and increased $\mathrm{Vd}$.

Miller et al found shorter $\mathrm{T}_{1 / 2}$ in three term infants and one preterm infant than in older children and adults after oral APAP. ${ }^{11}$ Other studies found longer $T_{1 / 2}$ after rectal APAP in infants compared with oral doses in adults. ${ }^{422} 23$ Our $T_{1 / 2}$ values were 11 hours in infants $28-32$ weeks of gestation and 4.8 hours in infants 32-36 weeks of gestation and were inversely related to gestational age. This agrees with the published findings, with values of 2.7 to 4.9 hours in term infants in the first month of life and of 1.0 to 2.4 hours up to the age of 1 year. ${ }^{10}$ Doses of $18 \mathrm{mg} / \mathrm{kg}$ or more produced therapeutic concentrations in the 28 to 32 week gestation group, and doses up to $26.6 \mathrm{mg} / \mathrm{kg}$ were given without any adverse effects. Because of the long $T_{1 / 2}$ we recommend that multiple doses should be given every 8 to 12 hours.

The pain scores we used ${ }^{12} 13$ did not correlate with the concentrations of APAP, probably because most of the infants scored low on the pain score indicating minimal or no pain. It may be that this pain score is not particularly suitable for measuring pain in preterm infants or that it is only suited for the assessment of acute severe pain. In the original study this pain score was used for older infants, ${ }^{12}$ and later modified for use in younger infants. ${ }^{13}$ Manne et al showed that in older children simultaneous assessment of pain by patients, nurses, and parents showed comparable results. ${ }^{25}$ Postgestational age seems to be important when considering the pain response of infants of less than 32 weeks of age. ${ }^{26}$

In several double blind cross over studies in healthy volunteers given normal therapeutic doses, analgesic effects occurred with concentrations $<10 \mathrm{mg} / 1 .{ }^{20}$ If the same applies for the infants in our study, they were having suitable pain management and the pain score was appropriate.

Exaggerated concerns for overdosing and hepatotoxicity, or increased bilirubin concentrations have been the main reasons for withholding APAP from neonates. Neonates are capable of metabolising APAP, not by glucuronidation but also by sulphation, ${ }^{1122} 27$ and there is no correlation with plasma bilirubin concentration. ${ }^{22}$

Limited data show that even when APAP reaches toxic concentrations in preterm neonates, detoxification by the mixed function oxidase system is sufficient, without any adverse effects. ${ }^{16}{ }^{17}$ Even when glucuronide and sulphate conjugation (phase II reaction) have reached their maximum capacity, this normally minor pathway metabolises APAP as long as glutathione is not depleted. Part of the APAP is not metabolised and excreted unchanged in the urine.

Our findings that urinary excretion was primarily as APAP-S, resulting in a low glucuronide to sulphate ratio, agree with the results of earlier studies. ${ }^{11} 1622$ The values in our groups show a considerable decrease in $\mathrm{G}: \mathrm{S}$ ratio with 
earlier gestational age. Fig 4 shows the comparison of G:S ratio in our study with two other studies in older infants, children, and adults. ${ }^{1122}$ Our results are supported by several studies showing decreased fetal and neonatal UDPG-T activity, ${ }^{28-30}$ and by the presence of a well developed sulphation pathway both in vivo $^{21}$ in term neonates and in vitro ${ }^{31}$ in fetal liver at 19-22 weeks of age. Furthermore, two other studies showed even higher sulphation rates than those of glucuronidation in children aged $7-10$ years, compared with adults. ${ }^{1132}$ Our results differ from those of a recent study in which 2 to 6 day old term infants ingested paracetamol in their mothers' breast milk in amounts of $0.3-18.5 \mathrm{mg} / \mathrm{kg}$, and excreted it mainly as APAP-G. ${ }^{33}$ However, in this study urine sampling only took place for 3 hours; in other words with the longer $T_{1 / 2}$ in infants a substantial part was not yet excreted and maximal recovery of the ingested dose was $4.4 \% .^{33}$

We found only minute amounts of APAP-C and no APAP-M in the urine. As APAP-M was found in urine of a preterm infant after maternal overdose, ${ }^{17}$ it might be that in preterm infants APAP-M is only formed after doses higher than our dose or after prolonged multiple doses, or that it is merely a reflection of their mothers' excretion pattern.

We conclude that rectally administered APAP is safe in preterm infants as single dose treatment. As prolonged rectal absorption from this dose is evident, a higher dose might be needed to shorten the interval to reach therapeutic concentrations. Prolonged rectal absorption and plasma clearance subsequent to developmental immaturity in the phase I and phase II pathways which govern APAP clearance were evident. Rectal APAP was apparently tolerated without any overt evidence of adverse effects.

Sulphation is the major metabolic pathway, while the G:S ratio is positively related with post conceptional age. Furthermore, our study provides new ways of using APAP: in preterm infants the choice is no longer strong analgesics or no analgesic, but an intermediate analgesic for moderate pain.

We gratefully acknowledge the contribution by the nurses from intensive care to the collection of the data, and Willem Grol for his statistical advice.

1 Anand KJS, Hickey PR. Pain and its effects in the human neonate and fetus. $N$ Engl f Med 1987;317:1321-9.

2 Hartley R, Green M, Quinn M, Levene MI. Pharmacokinetics of morphine infusion in premature neonates. Arch Dis Child 1993;69:55-8

3 Shannon M, Berde CB. Pharmacological management of pain in children and adolescents. Pediatr Clin North Am 1989;36:855-71.

4 Hopkins CS, Underhill S, Booker PD. Pharmacokinetics of paracetamol after cardiac surgery. Arch Dis Child paracetamol afte.

5 Morselli PL, Franco-Morselli R, Bossi L. Clinical pharmacokinetics in newborns and infants. Age related differences and therapeutic implications. Clin Pharmacokinet 1980;5:485-527.
6 Van den Anker JN, Schoemaker RC, Hop WCJ, et al. Ceftazidime pharmacokinetics in preterm infants: effect of renal function and gestational age. Clin Pharmacol Ther 1995;58:650-9.

7 McIntosh N. Pain in the newborn, a possible new starting point. Eur f Pediatr 1997;156:173-7.

8 Farr V, Mitchell RG, Neligan GA, Parkin JM. The definition of some external characteristics in the assessment of gestational age in the newborn infant. Dev Med Child Neurol 1966;8:507-11.

9 Stevens HM, Gill R. High performance liquid chromatography systems for the analysis of analgesic and non-steroidal phy systems for the analysis of analgesic and non-steroidal togr 1986;370:39-47.

10 Rumack BH. Aspirin versus acetaminophen: A comparative view. Pediatrics 1978;62:943-6.

11 Miller RP, Roberts RJ, Fischer LJ. Acetaminophen elimination kinetics in neonates, children, and adults. Clin Pharmacol Ther 1976;19:284-94.

12 McGrath PA, de Veber LL, Hearn MT. Multidimensional pain assessment in children. Adv Pain Res Ther 1985;9:387-93.

13 Consensus report. Prevention and treatment of acute pain in children. Dutch National Organization for Quality Assurance in Hospitals. Utrecht: CBO, 1993.

14 Norusis MJ. Statistical Package for the Social Sciences; SSPS users guide. New York: McGraw-Hill, 1983.

15 Penna A, Buchanan N. Paracetamol poisoning in children and hepatotoxicity. Br f Clin Pharmacol 1991;32:143-9.

16 Lederman S, Fysh WJ, Tredger M, Gamsu HR. Neonatal paracetamol poisoning; treatment by exchange transfuparacetamol poisoning; treatment
sion. Arch Dis Child 1983;58:631-3.

17 Roberts I, Robinson MJ, Mughal MZ, Ratcliffe JG, Prescott LF. Paracetamol metabolites in the neonate following maternal overdose. Br f Pharmacol 1984;18:201-6.

18 Lauterburg BH, Vaishnav Y, Stillwell WG, Mitchell JR. The effects of age and glutathione depletion on hepatic glutathione turnover in vivo determined by acetaminophen probe analysis. I Pharmacol Exp Ther 1980;213:54-8.

19 Adamson GM, Harman AW. A role for the gluthatione peroxidase/reduction system in the protection from paracetamol toxicity in isolated mouse hepatocytes. Biochem Pharm 1989;38:3323-30.

20 Moolenaar F, Schoonen AJM, Everts A, Huizinga T. Biopharmaceutics of rectal administration of drugs in man. 4. Absorption rate and bioavailability of paracetamol from fatty suppositories. Pharm Week [Sci] 1979;1:89-94.

21 Blumer JL, Reed MD. Principles of neonatal pharmacology. In: Yaffe SJ, Aranda JV, eds. Pediatric pharmacology. Philadelphia: WB Saunders Co, 1992: 164-77.

22 Levy G, Khanna NN, Soda DM, Tsuzuki O, Stern L. Pharmacokinetics of acetaminophen in the human neonate: formation of acetaminophen glucuronide and sulfate in formation of acetaminophen glucuronide and sulfate in relation to plasma bilirubin concentration
acid excretion. Pediatrics $1975 ; 55: 818-25$.

23 Rawlins MD, Henderson DB, Hijab AR. Pharmacokinetics of paracetamol (acetaminophen) after intravenous and oral administration. Eur f Clin Pharmacol 1977;11:283-6.

24 Prescott LF. Kinetics and metabolism of paracetamol and phenacetin. Br f Clin Pharmacol 1980;10:291S-8S.

25 Manne SL, Jacobsen PB, Redd WH. Assessment of acute pediatric pain: do child self-report, parent ratings, and nurse ratings measure the same phenomenon? Pain 1992;48:45-52.

26 Johnston CC, Stevens BJ, Yang F, Horton L. Differential response to pain by very premature neonates. Pain 1995;61:471-9.

27 Rumore MM, Blaiklock RG. Influence of age-dependent pharmacokinetics and metabolism on acetaminophen hepatotoxicity. F Pharm Sci 1992;81:203-7.

28 Felsher BF, Maiman JE, Carpio NM, VanCouvering K, Woolley MM. Reduced hepatic bilirubin uridine diphosphate glucuronyl transferase and uridine diphosphate gluphate glucuronyl transferase and uridine diphosphate glu1978;12:838-40.

29 Rane A, Sjöqvist F, Orrenius S. Drug and fetal metabolism. Clin Pharmacol Ther 1973;14:666-72.

30 Rane A, Tomson G. Prenatal and neonatal drug metabolism in man. Eur f Clin Pharmacol 1980;18:9-15.

31 Rollins DE, von Bahr C, Glaumann H, Moldéus P, Rane A. Acetaminophen: potentially toxic metabolite formed by human fetal and adult liver microsomes and isolated fetal liver cells. Science 1979;205:1414-6.

32 Alam SN, Roberts RJ, Fischer LJ. Age-related differences in salicylamide and acetaminophen conjugation in man. $f$ Pediatr 1977;90:130-5.

33 Notoriani LJ, Oldham HG, Bennett PN. Passage of paracetamol into breast milk and its subsequent metabolism by the neonate. Br f Clin Pharmacol 1987;24:63-7. 